\title{
Periodic Solutions with Minimal Period for Fourth-Order Nonlinear Difference Equations
}

\author{
Xia Liu $\mathbb{D}^{1,2}$ Tao Zhou, ${ }^{3}$ Haiping Shi, ${ }^{4,5}$ Yuhua Long, ${ }^{6,7}$ and Zongliang Wen ${ }^{8}$ \\ ${ }^{1}$ Oriental Science and Technology College, Hunan Agricultural University, Changsha 410128, China \\ ${ }^{2}$ Science College, Hunan Agricultural University, Changsha 410128, China \\ ${ }^{3}$ School of Business Administration, South China University of Technology, Guangzhou 510640, China \\ ${ }^{4}$ Modern Business and Management Department, Guangdong Construction Polytechnic, Guangzhou 510440, China \\ ${ }^{5}$ School of Mathematics and Statistics, Central South University, Changsha 410083, China \\ ${ }^{6}$ School of Mathematics and Information Science, Guangzhou University, Guangzhou 510006, China \\ ${ }^{7}$ Key Laboratory of Mathematics and Interdisciplinary Sciences of Guangdong Higher Education Institute, Guangzhou University, \\ Guangzhou 510006, China \\ ${ }^{8}$ College of Pharmacy, Guangxi University of Chinese Medicine, Nanning 530001, China
}

Correspondence should be addressed to Xia Liu; xia991002@163.com

Received 4 January 2018; Accepted 8 April 2018; Published 10 May 2018

Academic Editor: Guang Zhang

Copyright (C) 2018 Xia Liu et al. This is an open access article distributed under the Creative Commons Attribution License, which permits unrestricted use, distribution, and reproduction in any medium, provided the original work is properly cited.

A fourth-order nonlinear difference equation is considered. By making use of critical point theory, some new criteria are obtained for the existence of periodic solutions with minimal period. The main methods used are a variational technique and the Linking Theorem.

\section{Introduction}

Let $\mathbf{N}, \mathbf{Z}$, and $\mathbf{R}$ denote the sets of all natural numbers, integers, and real numbers, respectively. [.] denotes the greatest-integer function. For any $a, b \in \mathbf{Z}$, define $\mathbf{Z}(a, b)=$ $\{a, a+1, \ldots, b\}$ when $a \leq b . x^{*}$ denotes the transpose of a vector $x$.

Consider the following fourth-order nonlinear difference equation:

$$
\Delta^{4} u_{n-2}=f\left(n, u_{n}\right), \quad n \in \mathbf{Z},
$$

where $\Delta$ is the forward difference operator $\Delta u_{n}=u_{n+1}-u_{n}$; $\Delta^{i} u_{n}=\Delta\left(\Delta^{i-1} u_{n}\right)$ for $i \geq 2$, and $f \in C^{1}\left(\mathbf{R}^{2}, \mathbf{R}\right)$ and $f(n, u)$ are $T$-periodic in $n$ for a given positive integer $T$.

Equation (1) can be considered as a discrete analogue of continuous versions of problem like

$$
u^{(4)}(t)=f(t, u(t)), \quad t \in \mathbf{R},
$$

which is used to describe the stationary states of the deflection of an elastic beam [1]. Equations similar to (2) arise in the study of the existence of solutions to differential equations; we refer the reader to [2-7] and the references therein.

The theory of nonlinear difference equations has been widely used to study discrete models in many fields such as finance insurance, computing, electrical circuit analysis, dynamical systems, physical field, and biology. Because of their importance, many literature and monographs deal with their existence and uniqueness problems; see [8-27].

Using the critical point theory and monotone operator theory, $\mathrm{He}$ and $\mathrm{Su}$ [13] studied the following discrete nonlinear fourth-order boundary value problems:

$$
\begin{aligned}
\Delta^{4} u_{n-2}+\eta \Delta^{2} u_{n-1}-\xi u_{n}=\lambda f\left(n, u_{n}\right), & \\
n & \in \mathbf{Z}[a+1, b+1],
\end{aligned}
$$

with three parameters. Some existence, multiplicity, and nonexistence results of nontrivial solutions are obtained. 
Chen and Tang [12] in 2011 were concerned with the existence of infinitely many homoclinic orbits from 0 of the fourth-order difference system

$$
\Delta^{4} u_{n-2}+q_{n} u_{n}=f\left(n, u_{n+1}, u_{n}, u_{n-1}\right), \quad n \in \mathbf{Z}
$$

by using the symmetric Mountain Pass Lemma and established some existence criteria to guarantee that (4) has infinitely many homoclinic orbits.

In 2012, $\mathrm{Ma}$ and $\mathrm{Lu}$ [19] showed the existence and multiplicity of positive solutions of the nonlinear discrete fourth-order boundary value problem for

$$
\Delta^{4} u_{n-2}=\lambda h_{n} f\left(u_{n}\right), \quad n \in\{2,3, \ldots, T\},
$$

by using Dancer's global bifurcation theorem.

Liu et al. [18] studied the existence and multiplicity of periodic and subharmonic solutions to the following nonlinear difference equation:

$$
\Delta^{2}\left(r_{n-2} \Delta^{2} u_{n-2}\right)=f\left(n, u_{n+1}, u_{n}, u_{n-1}\right), \quad n \in \mathbf{Z},
$$

using variational technique and the Linking Theorem.

By employing the variational methods, Yu et al. [22] got some new criteria for the existence of subharmonic solutions with prescribed minimal period of second-order nonlinear difference equation

$$
\Delta^{2} u_{n-1}+A \sin u_{n}=f(n), \quad n \in \mathbf{Z} .
$$

Applying the direct method of the calculus of variations and the mountain pass technique, Leszczyński [17] in 2015 proved the existence of at least one and at least two solutions to the fourth-order discrete anisotropic boundary value problem with both advance and retardation of form

$$
\begin{array}{rl}
\Delta^{2}\left(\gamma_{n}-1 \phi_{p_{n}}\left(\Delta^{2} u_{n-2}\right)\right)=f\left(n, u_{n+1}, u_{n}, u_{n-1}\right) & \\
n & n \mathbf{Z}[1, k] .
\end{array}
$$

Nonexistence of nontrivial solutions was also obtained.

Motivated by the recent papers $[12,16]$, our purpose in this work is to apply Linking Theorem in critical point theory to establish some conditions for the nonlinear function $f$ which are able to guarantee the existence of at least two nontrivial periodic solutions with minimal period $m T$ for the above problem.

Throughout this paper, we suppose that $m$ is a given integer and $m>1$. Let

$$
\omega=\frac{2 \pi}{T}
$$

To wit, we get the following.

Theorem 1. Assume that the following hypotheses are satisfied:

$\left(F_{1}\right)$ there exists a function $F \in C^{2}\left(\mathbf{R}^{2}, \mathbf{R}\right)$ with $F(n+T, u)=$ $F(n, u), F(-n,-u)=F(n, u)$, and $F(n, u) \geq 0$ and it satisfies

$$
\frac{\partial F(n, u)}{\partial u}=f(n, u), \quad \forall n \in \mathbf{Z}
$$

$\left(F_{2}\right)$ there exist positive constants $\epsilon_{1}, \alpha \in\left(0,8 \sin ^{4}(\pi / m T)\right)$ such that

$$
F(n, u) \leq \alpha u^{2}, \quad \forall n \in \mathbf{Z},|u| \leq \epsilon_{1}
$$

$\left(F_{3}\right)$ there exist positive constants $\zeta, \beta \in(8,+\infty)$ such that

$$
F(n, u) \geq \beta u^{2}-\zeta, \quad \forall(n, u) \in \mathbf{Z} \times \mathbf{R}
$$

$\left(F_{4}\right)$ there exist positive constants $\rho$ and $\mu>v$ such that

$$
\begin{aligned}
& \left(\frac{\partial^{2} F(n, u)}{\partial u^{2}} \eta, \eta\right) \leq \mu \eta^{2}, \quad \forall(n, u) \in \mathbf{Z} \times \mathbf{R}, \eta \in \mathbf{R}, \\
& \left(\frac{\partial^{2} F(n, u)}{\partial u^{2}} \eta, \eta\right) \geq \nu \eta^{2}, \quad \forall|u| \leq \rho, n \in \mathbf{Z}, \eta \in \mathbf{R}
\end{aligned}
$$

$\left(F_{5}\right)$ if $u$ is a solution of (1) with minimal period $l T, l$ is a rational number, and $f(n, u)$ also has a minimal period $l T$, then $l$ must be an integer.

$\left(F_{6}\right)$ let $\tau_{m}$ be the least prime factor of $m$,

$$
\begin{aligned}
& \nu>16 \sin ^{4} \frac{\omega}{2 m}, \\
& 16 \sin ^{4} \frac{\omega \tau_{m}}{2 m}>\mu, \\
& \sum_{n=1}^{m T} f^{2}(n, 0) \\
& <\frac{\pi \rho^{2}\left(32 \sin ^{4}\left(\omega \tau_{m} / 2 m\right)-2 \mu\right)\left(2 \nu-32 \sin ^{4}(\omega / 2 m)\right)}{\omega} .
\end{aligned}
$$

Then (1) has at least two nontrivial periodic solutions with minimal period $m T$.

Theorem 2. Assume that $\left(F_{1}\right),\left(F_{4}\right)-\left(F_{6}\right)$, and the following conditions are satisfied:

$\left(F_{7}\right) \lim _{|u| \rightarrow 0}\left(F(n, u) / u^{2}\right)=0, \forall(n, u) \in \mathbf{Z} \times \mathbf{R}$;

$\left(F_{8}\right)$ there exist positive constants $\epsilon_{2}$ and $\gamma>2$ such that for $n \in \mathbf{Z}$ and $|u| \geq \epsilon_{2}$,

$$
0<\gamma F(n, u) \leq u f(n, u)
$$

Then (1) has at least two nontrivial periodic solutions with minimal period $m T$.

\section{Variational Structure and Some Lemmas}

Define the functional $J$ as follows:

$$
J(u):=\frac{1}{2} \sum_{n=1}^{m T}\left(\Delta^{2} u_{n-1}\right)^{2}-\sum_{n=1}^{m T} F\left(n, u_{n}\right),
$$

on the finite-dimensional Hilbert space

$$
E_{m T}=\left\{u \mid u_{n+m T}=u_{n}, \forall n \in \mathbf{Z}\right\},
$$


where

$$
u=\left(\ldots, u_{-n}, \ldots, u_{-1}, u_{0}, u_{1}, \ldots, u_{n}, \ldots\right) .
$$

For $m T>2$, define

$$
M:=\left(\begin{array}{cccccc}
2 & -1 & 0 & \cdots & 0 & -1 \\
-1 & 2 & -1 & \cdots & 0 & 0 \\
0 & -1 & 2 & \cdots & 0 & 0 \\
\cdots & \cdots & \cdots & \cdots & \cdots & \cdots \\
0 & 0 & 0 & \cdots & 2 & -1 \\
-1 & 0 & 0 & \cdots & -1 & 2
\end{array}\right)_{m T \times m T} .
$$

For $m T=2$, define

$$
M:=\left(\begin{array}{cc}
2 & -2 \\
-2 & 2
\end{array}\right) .
$$

The argument need not be changed and we omit it.

$J(u)$ can be rewritten as

$$
\begin{aligned}
J(u) & =\frac{1}{2} \sum_{n=1}^{m T}\left(\Delta^{2} u_{n}, \Delta^{2} u_{n}\right)-\sum_{n=1}^{m T} F\left(n, u_{n}\right) \\
& =\frac{1}{2} x^{*} M x-\sum_{n=1}^{m T} F\left(n, u_{n}\right),
\end{aligned}
$$

where $x=\left(\Delta u_{1}, \Delta u_{2}, \ldots, \Delta u_{m T}\right)^{*}$.

Clearly, $J \in C^{1}\left(E_{m T}, \mathbf{R}\right)$ and for any $u \in E_{m T}$, by using $u_{0}=u_{m T}, u_{1}=u_{m T+1}$, we can compute the partial derivative as

$$
\frac{\partial J}{\partial u_{n}}=\Delta^{4} u_{n-2}-f\left(n, u_{n}\right), \quad \forall n \in \mathbf{Z}(1, m T) .
$$

Therefore, $u \in E_{m T}$ is a critical point of $J$ on $E_{m T}$ if and only if

$$
\Delta^{4} u_{n-2}=f\left(n, u_{n}\right), \quad \forall n \in \mathbf{Z}(1, m T) .
$$

Since $u=\left\{u_{n}\right\}_{n \in \mathbf{Z}} \in E_{m T}$ and $f(n, u)$ in the first variable $n$ are $m T$-periodic in $n$, we can reduce the existence of periodic solutions of (1) to the existence of critical points of $J$ on $E_{m T}$.

By matrix theory, the eigenvalues of $M$ can be given by

$$
\lambda_{j}=4 \sin ^{2} \frac{j \pi}{m T}, \quad j=0,1,2, \ldots, m T-1 .
$$

It is easy to see that 0 is an eigenvalue of $M$ and $\lambda_{j}>0, j=$ $1,2, \ldots, m T-1$, and

$$
\begin{aligned}
& \min \left\{\lambda_{1}, \lambda_{2}, \ldots, \lambda_{m T-1}\right\}=4 \sin ^{2} \frac{\pi}{m T}, \\
& \max \left\{\lambda_{1}, \lambda_{2}, \ldots, \lambda_{m T-1}\right\} \leq 4 .
\end{aligned}
$$

Denote

$$
C=\operatorname{ker} M=\left\{u \in E_{m T} \mid M u=0 \in \mathbf{R}^{m T}\right\} .
$$

Therefore,

$$
C=\left\{u \in E_{m T} \mid u=\{\xi\}, \xi \in \mathbf{R}\right\} .
$$

Let $D$ be the direct orthogonal complement of $E_{m T}$ to $C$; that is, $E_{m T}=C \oplus D$.

For $1 \leq j \leq[(m T-1) / 2]$, the eigenvectors of $M$ corresponding to $\lambda_{j}$ are

$$
\begin{aligned}
& \xi_{j}=\left(\cos \frac{2 j \pi}{m T}, \cos \frac{2 j \pi \cdot 2}{m T}, \ldots, \cos \frac{2 j \pi \cdot m T}{m T}\right)^{*}, \\
& \zeta_{j}=\left(\sin \frac{2 j \pi}{m T}, \sin \frac{2 j \pi \cdot 2}{m T}, \ldots, \sin \frac{2 j \pi \cdot m T}{m T}\right)^{*} .
\end{aligned}
$$

When $m T$ is even, the eigenvector corresponding to 4 is $\xi=(-1,1,-1,1, \ldots,-1,1)^{*}$. Let $P=\operatorname{span}\{\xi\}, Q=$ $\operatorname{span}\left\{\xi_{j}, j=1,2, \ldots,[(m T-1) / 2]\right\}$, and $S=\operatorname{span}\left\{\zeta_{j}, j=\right.$ $1,2, \ldots,[(m T-1) / 2]\}$. Then $E_{m T}=C \oplus P \oplus Q \oplus S$. For any $u \in E_{m T}$,

$$
u_{n}=a+\sum_{j=1}^{[(m T-1) / 2]}\left(a_{j} \cos \frac{\omega j}{m} n+b_{j} \sin \frac{\omega j}{m} n\right),
$$

$\forall n \in \mathbf{Z}$

where $a, a_{j}$, and $b_{j}$ are constants.

When $m T$ is odd, $E_{m T}=C \oplus Q \oplus S$. For any $u \in E_{m T}$,

$$
\begin{aligned}
u_{n}= & a+(-1)^{n} b \\
& +\sum_{j=1}^{[(m T-1) / 2]}\left(a_{j} \cos \frac{\omega j}{m} n+b_{j} \sin \frac{\omega j}{m} n\right),
\end{aligned}
$$

$\forall n \in \mathbf{Z}$

where $a, b, a_{j}$, and $b_{j}$ are constants.

Denote

$$
\widetilde{E}_{m T}=\left\{u \in E_{m T} \mid u_{-n}=u_{n}, \forall n \in \mathbf{Z}\right\} .
$$

Then $\widetilde{E}_{m T}=S$ and

$$
u_{n}=\sum_{j=1}^{[(m T-1) / 2]} b_{j} \sin \frac{\omega j}{m} n, \quad \forall n \in \mathbf{Z} .
$$

Let $B_{r}$ denote the open ball in $E$ about 0 of radius $r$ and let $\partial B_{r}$ denote its boundary.

Lemma 3 (Linking Theorem [1]). Let $G$ be a real Banach space, $G=G_{1} \oplus G_{2}$, where $G_{1}$ is finite dimensional. Assume that $J \in C^{1}(G, \mathbf{R})$ satisfies the PS condition and

$\left(J_{1}\right)$ there exist constants $b>0$ and $\varrho>0$ such that $\left.J\right|_{\partial B_{e} \cap G_{2}} \geq b$;

$\left(J_{2}\right)$ there exists an $e \in \partial B_{1} \cap G_{2}$ and a constant $\varsigma \geq \varrho$ such that $\left.J\right|_{\partial \Phi} \leq 0$, where $\Phi=\left(\bar{B}_{\varsigma} \cap G_{1}\right) \oplus\{s e \mid 0<s<\varsigma\}$. 
Then J possesses a critical value $c \geq b$, where

$$
c=\inf _{h \in \Gamma} \sup _{u \in \Phi} J(h(u)),
$$

and $\Gamma=\left\{h \in C(\bar{\Phi}, G)|h|_{\partial \Phi}=I\right\}$, where I denotes the identity operator.

Lemma 4. Assume that the hypotheses $\left(F_{1}\right)-\left(F_{3}\right)$ are satisfied. Then the functional $J$ is bounded from above in $E_{m T}$.

Proof. According to $\left(F_{3}\right)$ and (21), for any $u \in E_{m T}$,

$$
\begin{aligned}
J(u) & =\frac{1}{2} x^{*} M x-\sum_{n=1}^{m T} F\left(n, u_{n}\right) \\
& \leq \frac{1}{2} x^{*} M x-\sum_{n=1}^{m T}\left(\beta u_{n}^{2}-\zeta\right) \\
& \leq 2\|x\|^{2}-\beta\|u\|^{2}+m T \zeta,
\end{aligned}
$$

where $x=\left(\Delta u_{1}, \Delta u_{2}, \ldots, \Delta u_{m T}\right)^{*}$. Since

$$
\|x\|^{2}=\sum_{n=1}^{m T}\left(u_{n+1}-u_{n}, u_{n+1}-u_{n}\right)=u^{*} M u \leq 4\|u\|^{2},
$$

we get

$$
J(u) \leq(8-\beta)\|u\|^{2}+m T \zeta \leq m T \zeta .
$$

The desired results are obtained.

Lemma 5. Assume that the hypotheses $\left(F_{1}\right)-\left(F_{3}\right)$ are satisfied. Then the functional $J$ satisfies the PS condition.

Proof. Let $\left\{J\left(u^{(k)}\right)\right\}$ be a bounded sequence from below, that is, there exists a constant $b_{1}>0$ such that

$$
-b_{1} \leq J\left(u^{(k)}\right), \quad \forall k \in \mathbf{N} .
$$

Due to the proof of Lemma 4 , it is obvious that

$$
-b_{1} \leq J\left(u^{(k)}\right) \leq(8-\beta)\left\|u^{(k)}\right\|^{2}+m T \zeta, \quad \forall k \in \mathbf{N} .
$$

Thus,

$$
\left\|u^{(k)}\right\|^{2} \leq \frac{b_{1}+m T \zeta}{\beta-8} .
$$

That is, $\left\{u^{(k)}\right\}$ is a bounded sequence in the finite-dimensional space $E_{m T}$. Consequently, it has a convergent subsequence. The proof is finished.

Lemma 6. If $u$ is a critical point of $J(u)$ on $\widetilde{E}_{m T}$, then $u$ is a critical point of $J(u)$ on $E_{m T}$.

In a similar fashion to the proof of Lemma 4 and the process in [22], we can prove Lemma 6. The detailed proof is omitted.

Set

$$
\Omega_{l}=-\frac{m}{32 \sin ^{4}(\omega l / 2 m)-2 \mu} \sum_{n=1}^{m T} f^{2}(n, 0) .
$$

Lemma 7. Assume that the hypotheses $\left(F_{4}\right)-\left(F_{6}\right)$ are satisfied. If $u$ is a critical point of $J(u)$ on $\widetilde{E}_{m T}, J(u)<\Omega_{\tau_{m}}$, then $u$ has minimal period $m T$.

Proof. If not, there is a positive integer $l$ such that $u$ has minimal period $m T / l$. By $\left(F_{5}\right), l \geq \tau_{m}$. For any $u \in \widetilde{E}_{m T}$,

$$
u_{n}=\sum_{j=1}^{[(m T-l) / 2 l]} b_{j} \sin \frac{\omega l j}{m} n
$$

and then

$$
\begin{aligned}
J(u) & =\frac{1}{2} x^{*} M x-\sum_{n=1}^{m T} F\left(n, u_{n}\right) \\
& \geq 2 \sin ^{2} \frac{\omega l}{2 m}\|x\|^{2}-\sum_{n=1}^{m T} F\left(n, u_{n}\right),
\end{aligned}
$$

where $x=\left(\Delta u_{1}, \Delta u_{2}, \ldots, \Delta u_{m T}\right)^{*}$. Since

$$
\begin{aligned}
\|x\|^{2} & =\sum_{n=1}^{m T}\left(u_{n+1}-u_{n}, u_{n+1}-u_{n}\right)=u^{*} M u \\
& \geq 4 \sin ^{2} \frac{\omega l}{2 m}\|u\|^{2},
\end{aligned}
$$

we get

$$
\begin{aligned}
J(u) \geq & 8 \sin ^{4} \frac{\omega l}{2 m}\|u\|^{2}-\left(\sum_{n=1}^{m T} f^{2}(n, 0)\right)^{1 / 2}\|u\| \\
& -\frac{\mu}{2}\|u\|^{2} \\
\geq & -\frac{1}{32 \sin ^{4}(\omega l / 2 m)-2 \mu} \sum_{n=1}^{m T} f^{2}(n, 0) \\
\geq & -\frac{m}{32 \sin ^{4}(\omega l / 2 m)-2 \mu} \sum_{n=1}^{m T} f^{2}(n, 0) .
\end{aligned}
$$

Then

$$
J(u) \geq \Omega_{l} \geq \Omega_{\tau_{m}},
$$

which contradicts $J(u)<\Omega_{\tau_{m}}$. The proof is complete.

\section{Proof of Results}

Proof of Theorem 1. It comes from Lemma 4 that the functional $J$ is bounded from above on $E_{m T}$.

Take

$$
k_{0}=\sup _{u \in E_{m T}} J(u)
$$

On one hand, there exists a sequence $\left\{u^{(k)}\right\}$ on $E_{m T}$ such that

$$
k_{0}=\lim _{k \rightarrow \infty} J\left(u^{(k)}\right) .
$$


On the other hand, from (36), we get

$$
J(u) \leq(8-\beta)\|u\|^{2}+m T \zeta \leq m T \zeta, \quad \forall u \in E_{m T} .
$$

Thus, $\lim _{\|u\| \rightarrow+\infty} J(u)=-\infty$, which implies that $\left\{u^{(k)}\right\}$ is bounded. Therefore, $\left\{u^{(k)}\right\}$ has a convergent subsequence, denoted by $\left\{u^{\left(k_{j}\right)}\right\}$. Set

$$
\bar{u}=\lim _{j \rightarrow+\infty} u^{\left(k_{j}\right)}
$$

Due to the continuity of $J(u)$, it is obvious that $J(\bar{u})=k_{0}$. That is, $\bar{u}$ is a critical point of $J$ on $E_{m T}$.

Assumption $\left(F_{2}\right)$ implies that, for any $u \in D,\|u\| \leq \epsilon_{1}$,

$$
\begin{aligned}
J(u) & =\frac{1}{2} x^{*} M x-\sum_{n=1}^{m T} F\left(n, u_{n}\right) \geq \frac{1}{2} x^{*} M x-\alpha \sum_{n=1}^{m T} u_{n}^{2} \\
& \geq 2 \sin ^{2} \frac{\pi}{m T}\|x\|^{2}-\alpha\|u\|^{2},
\end{aligned}
$$

where $x=\left(\Delta u_{1}, \Delta u_{2}, \ldots, \Delta u_{m T}\right)^{*}$. Since

$$
\begin{aligned}
\|x\|^{2} & =\sum_{n=1}^{m T}\left(u_{n+1}-u_{n}, u_{n+1}-u_{n}\right)=u^{*} M u \\
& \geq 4 \sin ^{2} \frac{\pi}{m T}\|u\|^{2},
\end{aligned}
$$

then

$$
J(u) \geq\left(8 \sin ^{4} \frac{\pi}{m T}-\alpha\right)\|u\|^{2} .
$$

Let $\sigma=\left(8 \sin ^{4}(\pi / m T)-\alpha\right) \epsilon_{1}^{2}$. Therefore, the functional

$$
\left.J\right|_{\partial B_{\epsilon_{1}} \cap D} \geq \sigma
$$

Thus, we have proved that $k_{0}=\sup _{u \in E_{m T}} J(u) \geq \sigma>0$. At the same time, we have also proved that there exist constants $\sigma>0$ and $\epsilon_{1}>0$ such that $\left.J\right|_{\partial B_{\epsilon_{1}} \cap D} \geq \sigma$.

By $\sum_{n=1}^{m T}\left(\Delta^{2} u_{n-1}\right)^{2}=0, \forall u \in C$ and assumption $\left(F_{1}\right)$,

$$
J(u)=\frac{1}{2} \sum_{n=1}^{m T}\left(\Delta^{2} u_{n-1}\right)^{2}-\sum_{n=1}^{m T} F\left(n, u_{n}\right)=-\sum_{n=1}^{m T} F\left(n, u_{n}\right)
$$

$$
\leq 0 \text {. }
$$

Then $\bar{u} \notin C$ and the critical point $\bar{u}$ of $J$ corresponding to the critical value $c_{0}$ is a nontrivial periodic solution of (1).

Let $v \in \partial B_{1} \cap D$. Then, for any $\psi \in C$ and $t \in \mathbf{R}$, let $u=t v+\psi$. Then

$$
\begin{aligned}
J(u) & =\frac{1}{2} \sum_{n=1}^{m T}\left(\Delta^{2} u_{n}, \Delta^{2} u_{n}\right)-\sum_{n=1}^{m T} F\left(n, u_{n}\right) \\
& \leq \frac{t^{2}}{2} \sum_{n=1}^{m T}\left(\Delta^{2} v_{n}, \Delta^{2} v_{n}\right)-\sum_{n=1}^{m T} F\left(n, t v_{n}+\psi_{n}\right) \\
& \leq \frac{t^{2}}{2} \Upsilon^{*} M \Upsilon-\sum_{n=1}^{m T}\left[\beta\left(t v_{n}+\psi_{n}\right)^{2}-\zeta\right]
\end{aligned}
$$

$$
\begin{aligned}
& \leq 2 t^{2}\|\Upsilon\|^{2}-\beta \sum_{n=1}^{m T}\left(t v_{n}+\psi_{n}\right)^{2}+m T \zeta \\
& =2 t^{2}\|\Upsilon\|^{2}-\beta t^{2}-\beta\|\psi\|^{2}+m T \zeta,
\end{aligned}
$$

where $\Upsilon=\left(\Delta v_{1}, \Delta v_{2}, \ldots, \Delta v_{m T}\right)^{*}$. Since

$$
\|\Upsilon\|^{2}=\sum_{n=1}^{m T}\left(v_{n+1}-v_{n}, v_{n+1}-v_{n}\right)=v^{*} M v \leq 4,
$$

then

$$
J(u) \leq(8-\beta) t^{2}-\beta\|\psi\|^{2}+m T \zeta \leq-\beta\|\psi\|^{2}+m T \zeta .
$$

Therefore, there is a constant $\chi>\epsilon_{1}>0$ such that, for any $u \in \partial \Phi, J(u) \leq 0$, where $\Phi=\left(\bar{B}_{\chi} \cap C\right) \oplus\{t v \mid 0<t<\chi\}$. By the Linking Theorem, $J$ possesses a critical value $c \geq \sigma>0$, where

$$
c=\inf _{h \in \Gamma} \sup _{u \in \Phi} J(h(u))
$$

and $\Gamma=\left\{h \in C\left(\bar{\Phi}, E_{m T}\right)|h|_{\partial \Phi}=I\right\}$.

Similar to the proof of [18], we can prove that (1) has at least two $m T$-periodic nontrivial solutions. For simplicity, its proof is omitted.

By $\left(F_{4}\right)$, for $|u| \leq \rho$, we have

$$
\begin{aligned}
F(n, u) & =f(n, 0) u+\frac{1}{2} \frac{\partial^{2} F(n, \theta u)}{\partial u^{2}} u^{2} \\
& \geq f(n, 0) u+\frac{v}{2} u^{2}
\end{aligned}
$$

Then, for $|u| \leq \rho$,

$$
\begin{aligned}
J(u) & =\frac{1}{2} \sum_{n=1}^{m T}\left(\Delta^{2} u_{n}, \Delta^{2} u_{n}\right)-\sum_{n=1}^{m T} F\left(n, u_{n}\right) \\
& \leq \frac{1}{2} \sum_{n=1}^{m T}\left(\Delta^{2} u_{n}, \Delta^{2} u_{n}\right)-\frac{\nu}{2} \sum_{n=1}^{m T} u_{n}^{2}-\sum_{n=1}^{m T} f(n, 0) u_{n} .
\end{aligned}
$$

Take $u_{n}=\rho \sin (\omega / m) n$. From $\left(F_{1}\right)$, we have

$$
f(n, 0)=\sum_{j=1}^{[(T-1) / 2]} a_{j} \sin \frac{2 j \pi}{T} n=\sum_{j=1}^{[(T-1) / 2]} a_{j} \sin \frac{2 j \pi}{m T} m n,
$$

where $a_{j}$ is a constant. Note that $m>1$; we get

$$
\begin{aligned}
\sum_{n=1}^{m T} f(n, 0) u_{n} & =\sum_{j=1}^{[(T-1) / 2]} \rho a_{j} \sum_{n=1}^{m T} \sin \frac{2 j \pi}{m T} m n \cdot \sin \frac{2 \pi}{m T} n \\
& =0
\end{aligned}
$$

Therefore, we have

$$
J(u) \leq\left(32 \sin ^{4} \frac{\omega}{2 m}-2 v\right)\|u\|^{2} .
$$


Since

$$
\|u\|=\rho\left(\frac{m \pi}{\omega}\right)^{1 / 2}
$$

then

$$
J(u)=\frac{\left(32 \sin ^{4}(\omega / 2 m)-2 \nu\right) \rho^{2} m \pi}{\omega}<\Omega_{\tau_{m}} .
$$

It comes from Lemma 7 that the desired result is obtained.

Remark 8. Similarly to the above argument, we can also prove Theorem 2. For simplicity, we omit its proof.

\section{Conflicts of Interest}

The authors declare that they have no conflicts of interest.

\section{Acknowledgments}

This project is supported by the National Natural Science Foundation of China (no. 11501194) and Philosophy and Social Sciences Planning Research Project in Guangxi (no. 15FGL008). This work was carried out while visiting Central South University. The author Haiping Shi wishes to thank Professor Xianhua Tang for his invitation.

\section{References}

[1] P. H. Rabinowitz, Minimax Methods in Critical Point Theory with Applications to Differential Equations, vol. 65, American Mathematical Society, Providence, RI, USA, 1986.

[2] C. Guo, D. O'Regan, C. Wang, and R. P. Agarwal, "Existence of homoclinic orbits of superquadratic second-order Hamiltonian systems," Journal of Analysis and Its Applications, vol. 34, no. 1, pp. 27-41, 2015.

[3] C. Guo, D. O'Regan, Y. Xu, and R. P. Agarwal, "Existence of periodic solutions for a class of second-order superquadratic delay differential equations," Dynamics of Continuous, Discrete \& Impulsive Systems. Series A. Mathematical Analysis, vol. 21, no. 5, pp. 405-419, 2014.

[4] C. Guo, D. O’Regan, Y. Xu, and R. P. Agarwal, "Existence of homoclinic orbits of a class of second-order differential difference equations," Dynamics of Continuous, Discrete \& Impulsive Systems. Series B. Applications \& Algorithms, vol. 20, no. 6, pp. 675-690, 2013.

[5] C. J. Guo, D. ORegan, Y. T. Xu, and R. P. Agarwal, "Existence and multiplicity of homoclinic orbits of a second-order differential difference equation via variational methods," Applied Mathematics and Mechanics, vol. 4, no. 1, pp. 1-15, 2012.

[6] X. Tang and S. Chen, "Ground state solutions of NehariPohozaev type for Schrödinger-POIsson problems with general potentials," Discrete and Continuous Dynamical Systems - Series A, vol. 37, no. 9, pp. 4973-5002, 2017.

[7] X. H. Tang and S. Chen, "Ground state solutions of NehariPohozaev type for Kirchhoff-type problems with general potentials," Calculus of Variations and Partial Differential Equations, vol. 56, no. 4, pp. 110-134, 2017.
[8] Z. AlSharawi, J. M. Cushing, and S. Elaydi, Theory and Applications of Difference Equations and Discrete Dynamical Systems, Springer, New York, NY, USA, 2014.

[9] X. Cai, J. Yu, and Z. Guo, "Existence of periodic solutions for fourth-order difference equations," Computers \& Mathematics with Applications, vol. 50, no. 1-2, pp. 49-55, 2005.

[10] P. Chen and C. Tian, "Infinitely many solutions for SchrödingerMaxwell equations with indefinite sign subquadratic potentials," Applied Mathematics and Computation, vol. 226, pp. 492502, 2014.

[11] P. Chen, X. He, and X. H. Tang, "Infinitely many solutions for a class of fractional Hamiltonian systems via critical point theory," Mathematical Methods in the Applied Sciences, vol. 39, no. 5, pp. 1005-1019, 2016.

[12] P. Chen and X. H. Tang, "Existence of infinitely many homoclinic orbits for fourth-order difference systems containing both advance and retardation," Applied Mathematics and Computation, vol. 217, no. 9, pp. 4408-4415, 2011.

[13] T. He and Y. Su, "On discrete fourth-order boundary value problems with three parameters," Journal of Computational and Applied Mathematics, vol. 233, no. 10, pp. 2506-2520, 2010.

[14] M. Huang and Z. Zhou, "Ground state solutions of the periodic discrete coupled nonlinear Schrödinger equations," Mathematical Methods in the Applied Sciences, vol. 38, no. 8, pp. 1682-1695, 2015.

[15] M. Huang and Z. Zhou, "Standing wave solutions for the discrete coupled nonlinear Schrödinger equations with unbounded potentials," Abstract and Applied Analysis, vol. 2013, Article ID 842594, 6 pages, 2013.

[16] M. Huang and Z. Zhou, "On the existence of ground state solutions of the periodic discrete coupled nonlinear schrödinger lattice," Journal of Applied Mathematics, vol. 2013, Article ID 404369, 8 pages, 2013.

[17] M. Leszczyński, "Fourth-order discrete anisotropic boundaryvalue problems," Electronic Journal of Differential Equations, vol. 2015, no. 238, pp. 1-14, 2015.

[18] X. Liu, Y. Zhang, H. Shi, and X. Deng, "Periodic and subharmonic solutions for fourth-order nonlinear difference equations," Applied Mathematics and Computation, vol. 236, pp. 613620, 2014.

[19] R. Ma and Y. Lu, "Existence and multiplicity of positive solutions of a nonlinear discrete fourth-order boundary value problem," Abstract and Applied Analysis, vol. 2012, Article ID 918082, 17 pages, 2012.

[20] X. H. Tang, "Non-Nehari manifold method for periodic discrete superlinear Schrödinger equation," Acta Mathematica Sinica, vol. 32, no. 4, pp. 463-473, 2016.

[21] X. H. Tang and X. Lin, "Infinitely many homoclinic orbits for discrete Hamiltonian systems with subquadratic potential," Journal of Difference Equations and Applications, vol. 19, no. 5, pp. 796-813, 2013.

[22] J. S. Yu, Y. H. Long, and Z. M. Guo, "Subharmonic solutions with prescribed minimal period of a discrete forced pendulum equation," Journal of Dynamics and Differential Equations, vol. 16, no. 2, pp. 575-586, 2004.

[23] Z. Zhou and D. Ma, "Multiplicity results of breathers for the discrete nonlinear Schrödinger equations with unbounded potentials," Science China Mathematics, vol. 58, no. 4, pp. 781790, 2015.

[24] Z. Zhou and M. Su, "Boundary value problems for 2 n-order $\phi c$-Laplacian difference equations containing both advance and retardation," Applied Mathematics Letters, vol. 41, pp. 7-11, 2015. 
[25] Z. Zhou and J. S. Yu, "Homoclinic solutions in periodic nonlinear difference equations with superlinear nonlinearity," Acta Mathematica Sinica, vol. 29, no. 9, pp. 1809-1822, 2013.

[26] Z. Zhou and J. Yu, "On the existence of homoclinic solutions of a class of discrete nonlinear periodic systems," Journal of Differential Equations, vol. 249, no. 5, pp. 1199-1212, 2010.

[27] Z. Zhou, J. Yu, and Y. Chen, "Homoclinic solutions in periodic difference equations with saturable nonlinearity," Science China Mathematics, vol. 54, no. 1, pp. 83-93, 2011. 


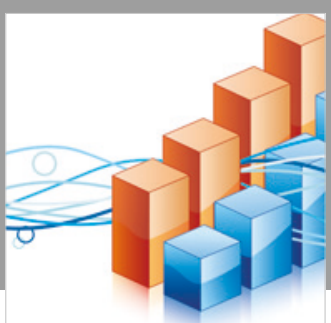

Advances in

Operations Research

\section{-n-m}
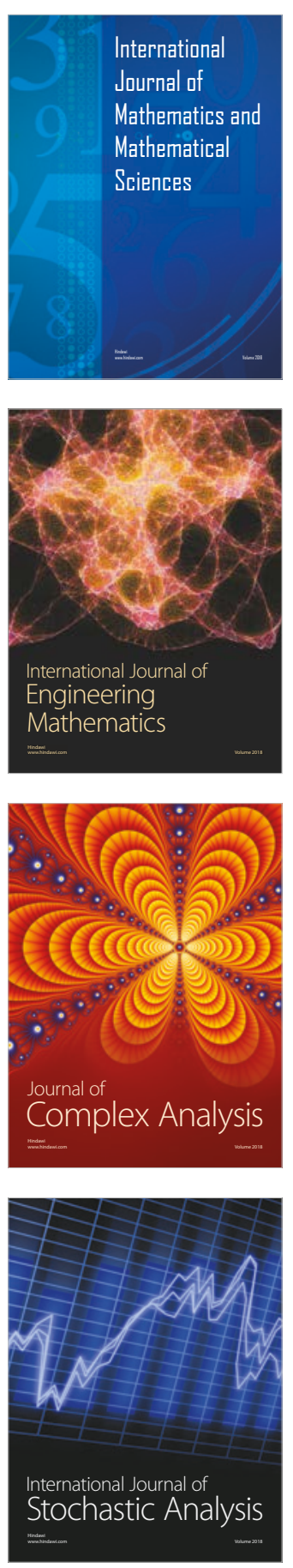
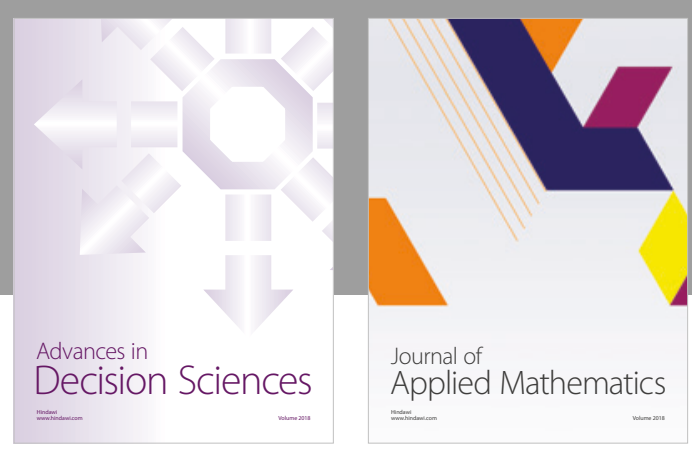

Journal of

Applied Mathematics
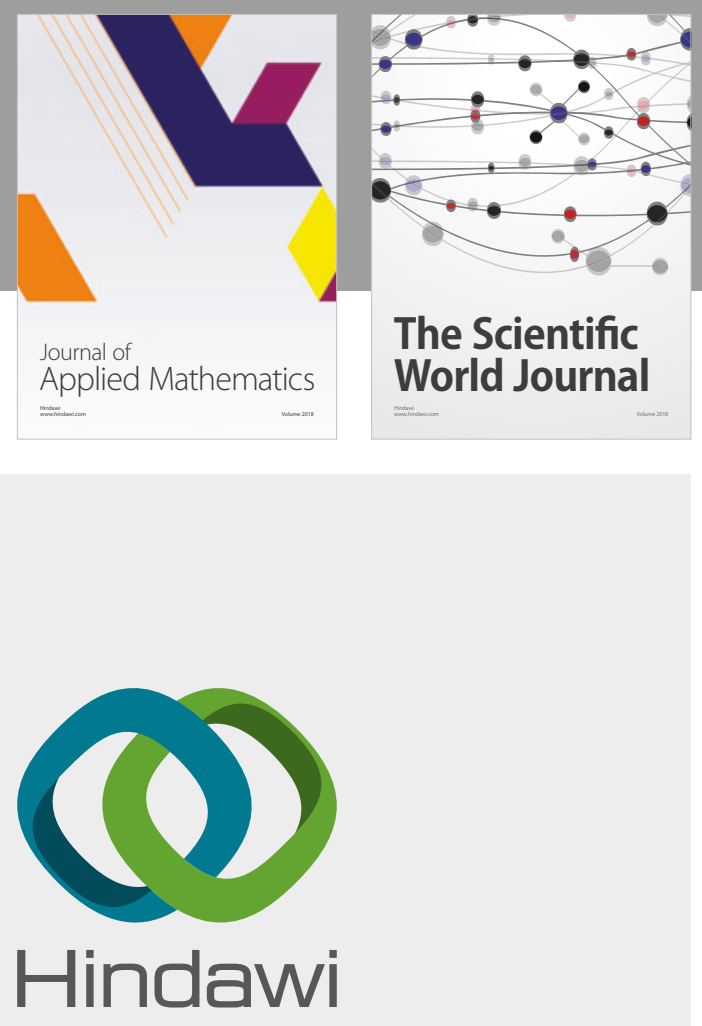

Submit your manuscripts at

www.hindawi.com

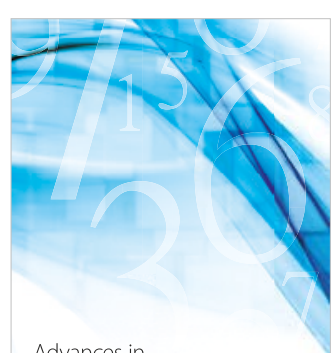

Advances in
Numerical Analysis
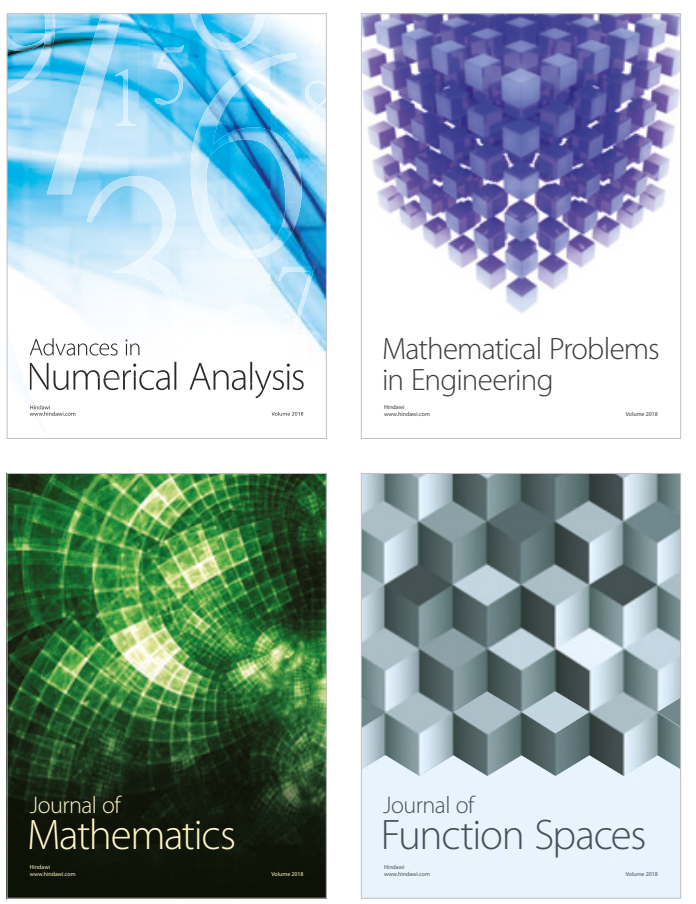

Mathematical Problems in Engineering

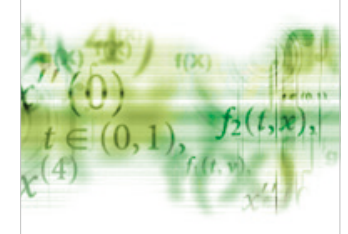

International Journal of

Differential Equations

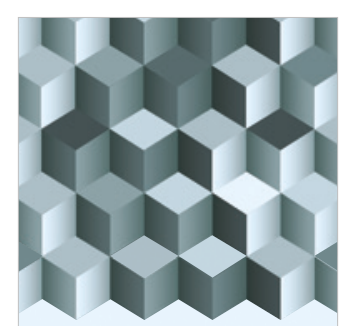

Journal of

Function Spaces

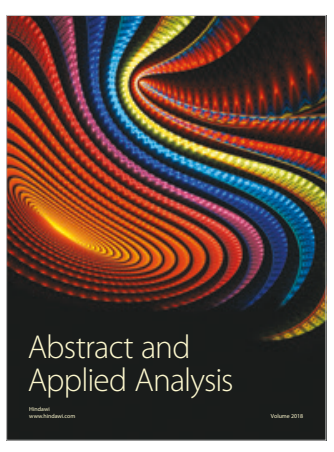

The Scientific

World Journal

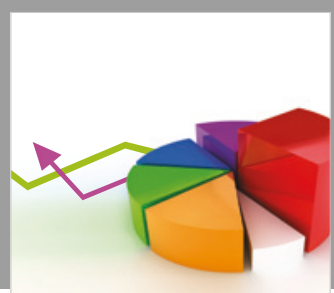

Journal of

Probability and Statistics
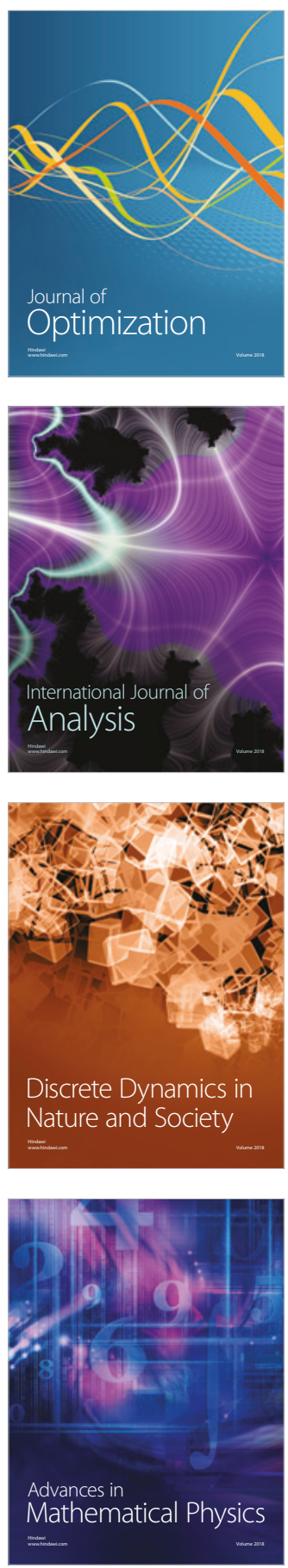